\title{
Fatal Invasive Pulmonary Aspergillosis in COVID-19 Patient with Acute Myeloid Leukemia in Iran
}

\author{
Elahe Nasri (D) Parisa Shoaei • Bahareh Vakili • Hossein Mirhendi • \\ Somayeh Sadeghi · Somayeh Hajiahmadi • Alireza Sadeghi • Afsane Vaezi (D) \\ Hamid Badali (1) · Hamed Fakhim (ㄷ)
}

Received: 4 July 2020/ Accepted: 19 September 2020/Published online: 3 October 2020

(C) Springer Nature B.V. 2020

\begin{abstract}
Although patients with severe immunodeficiency and hematological malignancies has been considered at highest risk for invasive fungal infection, patients with severe pneumonia due to influenza, and severe acute respiratory syndrome coronavirus (SARS-CoV) are also at a higher risk of developing invasive pulmonary aspergillosis (IPA). Recently, reports of IPA have also emerged among SARSCoV-2 infected patients admitted to intensive care units (ICUs). Here, we report a fatal case of probable
\end{abstract}

Handling Editor: Abdullah Mohammed Said Al-Hatmi.

E. Nasri · B. Vakili · S. Sadeghi · H. Fakhim $(\bowtie)$ Infectious Diseases and Tropical Medicine Research Center, Isfahan University of Medical Sciences, Isfahan, Iran

e-mail: Fakhiim.hamed@gmail.com

P. Shoaei

Nosocomial Infection Research Center, Isfahan University of Medical Sciences, Isfahan, Iran

H. Mirhendi

Research Core Facility Lab, Isfahan University of Medical Sciences, Isfahan, Iran

S. Sadeghi · A. Sadeghi

Department of Internal Medicine, School of Medicine, Isfahan University of Medical Sciences, Isfahan, Iran

\section{S. Hajiahmadi}

Department of Radiology, School of Medicine, Isfahan

University of Medical Sciences, Isfahan, Iran
IPA in an acute myeloid leukemia patient co-infected with SARS-CoV-2 and complicated by acute respiratory distress syndrome (ARDS). Probable IPA is supported by multiple pulmonary nodules with ground glass opacities which indicate halo sign and positive serum galactomannan results. Screening studies are needed to evaluate the prevalence of IPA in immunocompromised patients infected with SARS-CoV-2. Consequently, testing for the presence of Aspergillus in lower respiratory secretions and galactomannan in consecutive serum samples of COVID-19 patients with timely and targeted antifungal therapy based on

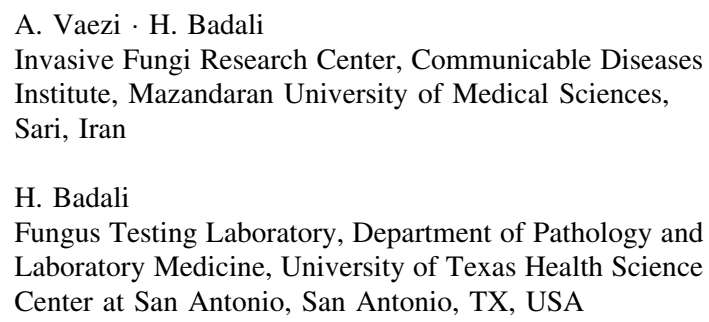
Laboratory Medicine, University of Texas Health Science Center at San Antonio, San Antonio, TX, USA 
early clinical suspicion of IPA are highly recommended.

Keywords COVID-19 - SARS-CoV-2 .

Aspergillosis · Acute myeloid leukemia

\section{Introduction}

Invasive fungal infections (IFIs) are life-threatening opportunistic infections, and immunosuppressed patients those with hematological malignancies have been considered at highest risk for developing IFIs [1-6]. Serious viral pulmonary infections are associated with increased risk for superinfections, including invasive pulmonary aspergillosis (IPA) in immunocompromised patients, particularly in those with hematological malignancies $[1,2]$. The prevalence of IPA in critically ill patients with acute respiratory distress syndrome (ARDS) is approximately $23 \%$ and an estimated mortality rate of more than $50 \%[1,3]$. Studies have revealed that influenza A and respiratory syncytial virus (RSV) were the most prevalent coinfecting respiratory viruses, and patients with viral co-infections have higher mortality rates than those with IPA alone[3, 6, 7]. Corona virus disease 2019 (COVID-19), caused by SARS-CoV-2, arose in late 2019 with rapid global spread. There have also been a number of reports of patients with probable or possible COVID-19 associated pulmonary aspergillosis $[1-4,8]$. Respiratory alveolar damage and abnormal mucociliary clearance caused by this virus may facilitate fungal invasion [2], and half of the IPA/ SARS-CoV-2 co-infections occur in non-neutropenic hospitalized patients in the intensive care unit (ICU) [2]. However, reports such co-infections in patients with hematologic malignancies are currently limited. Here, we report a case of COVID-19 pneumonia in an acute myeloid leukemia patient with probable invasive pulmonary aspergillosis.

\section{Case Report}

In March 2020, a 42-year-old female with a history of diabetes was hospitalized (day 0) in the HematologyOncology ward, Omid hospital, Isfahan, Iran, with newly diagnosed non-M3 acute myeloid leukemia
(AML), and began standard combination chemotherapy with idarubicin and cytarabine (day 2). She also received metformin $500 \mathrm{mg} /$ day for diabetes. Laboratory results prior to chemotherapy showed leukocytosis, anemia, and thrombocytopenia (Table 1). Following chemotherapy, she became febrile with a dry cough, dyspnea, and myalgia which lasted 2 days without hemoptysis, coryza, sore throat, pleuritic chest pain, or cyanosis (day 6). She complained of no other symptoms, nor had a history of travel or contact with known or suspected COVID-19 patients. However, she had been hospitalized for the prior two weeks due to weakness and anal bleeding. Physical examination (day 8) revealed a body temperature of $38.7{ }^{\circ} \mathrm{C}$, blood pressure of 100/70 $\mathrm{mm} \mathrm{Hg}$, a pulse of 109 beats per minute, respiratory rate of 22 breaths per minute, and oxygen saturation of $91 \%$ while the patient was breathing ambient air. The patient had some red spots and petechiae on the soft palate and both legs. Supplemental oxygen, delivered by nasal cannula at $4 \mathrm{~L}$ per minute was administered. Lung auscultation revealed rhonchi and examination of her heart revealed no murmurs, rubs, or gallops. The neurological examination and abdominal examination was unremarkable. Nasopharyngeal and oropharyngeal swabs were obtained; however, nucleic acid amplification test for influenza A and B was negative. Realtime reverse-transcriptase-polymerase-chain-reaction (rRT-PCR) by Roter-Gene Q 6000 (Qiagen, Hilden, Germany) for SARS-CoV-2 targeting the $\mathrm{N}$ gene and ORF1ab gene (Sansure, China) was positive. Blood and urine cultures were sterile. An arterial blood gas demonstrated a $\mathrm{pH}$ of $7.55, \mathrm{PaO}_{2} 49 \mathrm{~mm} \mathrm{Hg}, \mathrm{PCO}_{2}$ $38.6 \mathrm{~mm} \mathrm{Hg}, \mathrm{HCO}_{3} 29.3 \mathrm{mmol} / \mathrm{L}$ and the patient underwent oxygen supplementation. The high-resolution chest-computed tomography (CT) scan on day 8 demonstrated multiple vessel-related nodular opacities with grand glass halo including central and peripheral distribution, bilateral pleural effusion (Fig. 1). The patient was rehydrated with approximately $2 \mathrm{~L}$ of normal saline over the first 2 days of hospitalization. Following chemotherapy, laboratory results were notable for persistent leukopenia, anemia, and thrombocytopenia (Table 2). Empirical antibacterial therapy consisting of linezolid $600 \mathrm{mg}$ bid plus meropenem $3 \mathrm{~g} / \mathrm{d}$, lopinavir/ritonavir $400 / 100 \mathrm{mg}$ bid, and interferon Beta-1b $0.25 \mathrm{mg}$ SQ every $48 \mathrm{~h}$ for three dosages was started. On day 9 the nasal cannula was changed to non-invasive positive pressure 
Table 1 First admission clinical laboratory results

\begin{tabular}{|c|c|c|c|}
\hline Measure & $\begin{array}{l}\text { Reference } \\
\text { range }\end{array}$ & Hospital day 1 & Hospital day 2 \\
\hline White-cell count $($ per $\mu \mathrm{l})$ & $4000-11,000$ & 79,400 & 78,300 \\
\hline Red-cell count (per $\mu \mathrm{l}$ ) & $4,500,000-5,900,000$ & $2,120,000$ & $2,040,000$ \\
\hline Absolute neutrophil count (per $\mu \mathrm{l}$ ) & $1900-8000$ & & \\
\hline Absolute lymphocyte count (per $\mu \mathrm{l}$ ) & $900-5200$ & - & - \\
\hline Platelet count (per $\mu \mathrm{l}$ ) & $150,000-400,000$ & 18,000 & 33,000 \\
\hline Hemoglobin $(\mathrm{g} / \mathrm{dl})$ & $14-17.5$ & 6.6 & 6.2 \\
\hline Hematocrit (\%) & $41.5-50$ & 19.4 & 18.6 \\
\hline Sodium (mmol/liter) & $136-145$ & 127 & 128 \\
\hline Potassium (mmol/liter) & $3.5-5.1$ & 3 & 3.1 \\
\hline Blood urea nitrogen $(\mathrm{mg} / \mathrm{dl})$ & $8.4-25.7$ & 8 & 8 \\
\hline Creatinine (mg/dl) & $0.9-1.4$ & 0.8 & 0.8 \\
\hline Total bilirubin (mg/dl) & $0.1-1.1$ & 1 & 1 \\
\hline Alanine aminotransferase (U/liter) & $10-33$ & 10 & 10 \\
\hline Aspartate aminotransferase (U/liter) & $10-33$ & 15 & 15 \\
\hline Alkaline phosphatase (ALP) (U/liter) & 64-306 & 60 & 65 \\
\hline Prothrombin time (sec) & $11-15$ & 14.3 & 1403 \\
\hline International normalized ratio & $0.9-1.2$ & 1.12 & 1.14 \\
\hline erythrocyte sedimentation rate $(\mathrm{mm})$ & $0-20$ & 110 & - \\
\hline C-reactive protein (CRP) (mg/dl) & $1-6$ & 150 & - \\
\hline Ferritin (ng/ml) & $10-291$ & - & 1476 \\
\hline Lactate dehydrogenase (U/L) & $100-480$ & - & 780 \\
\hline D-dimer (ng/ml) & Positive; $>200$ & - & $>10,000$ \\
\hline Fibrinogen & $200-400$ & - & 272 \\
\hline Fibrin degradation product (FDP) $(\mu \mathrm{g} / \mathrm{ml})$ & Negative $;<5$ & - & 45 \\
\hline Blood group & $\mathrm{A}+$ & & \\
\hline
\end{tabular}
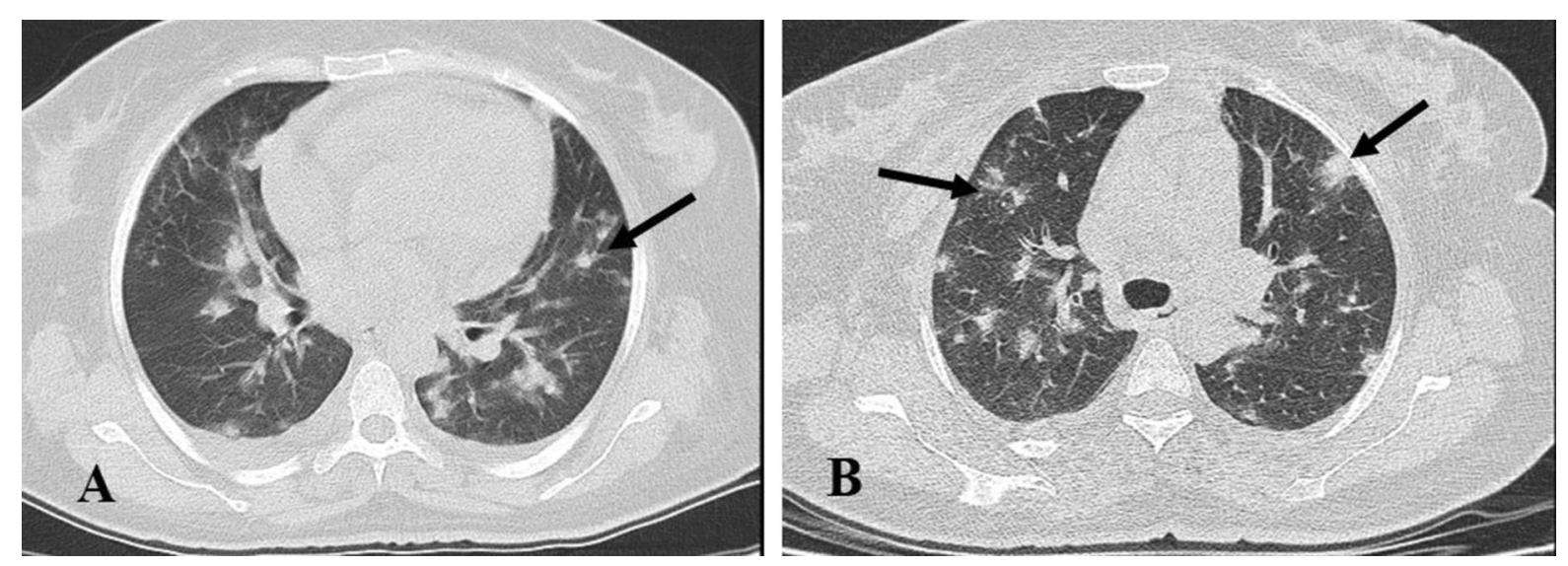

Fig. 1 a, b Chest CT scan revealed multiple vessel-related nodular opacities with grand glass halo with central and peripheral distribution, bilateral pleural effusion 


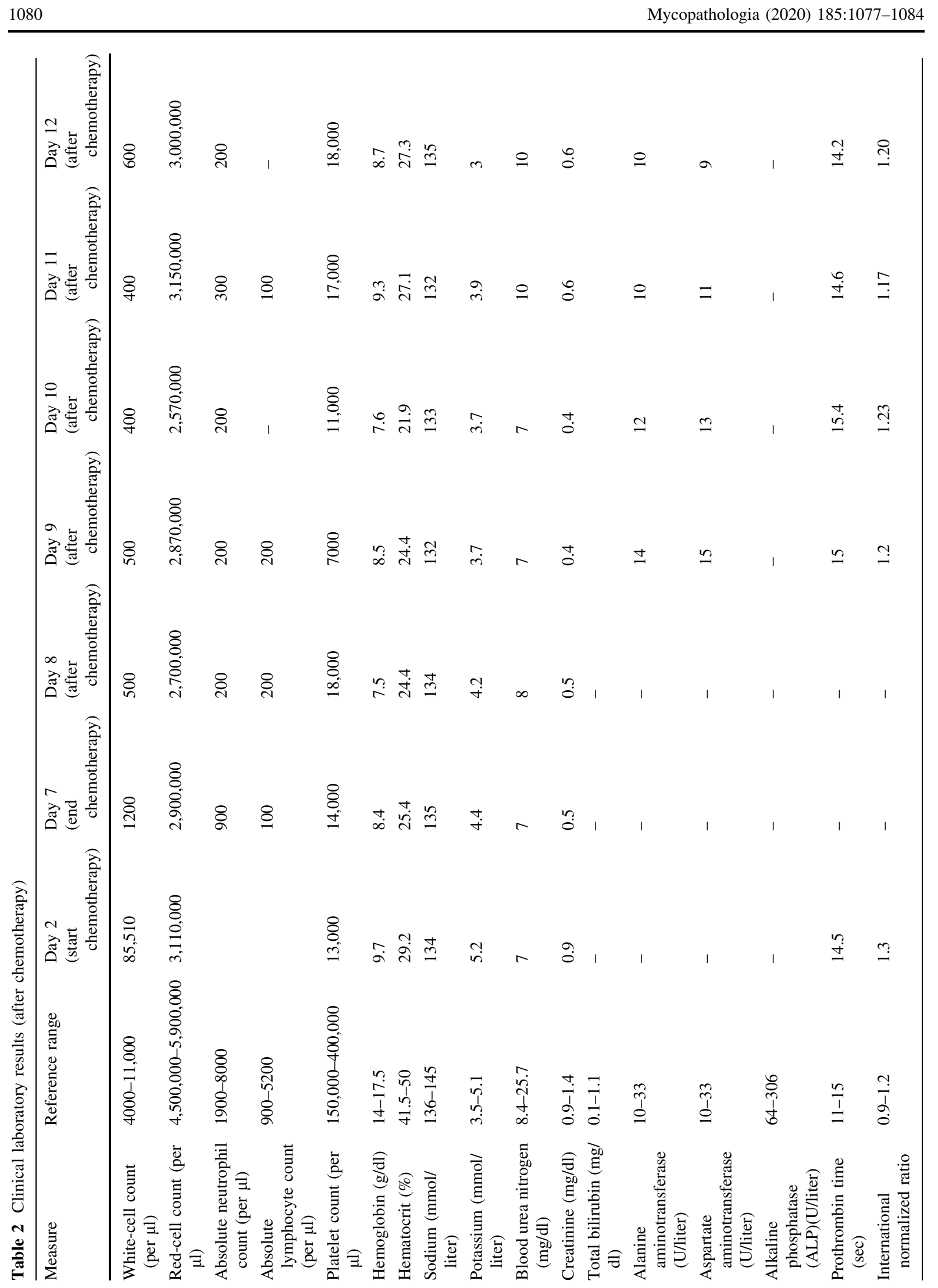

Springer 


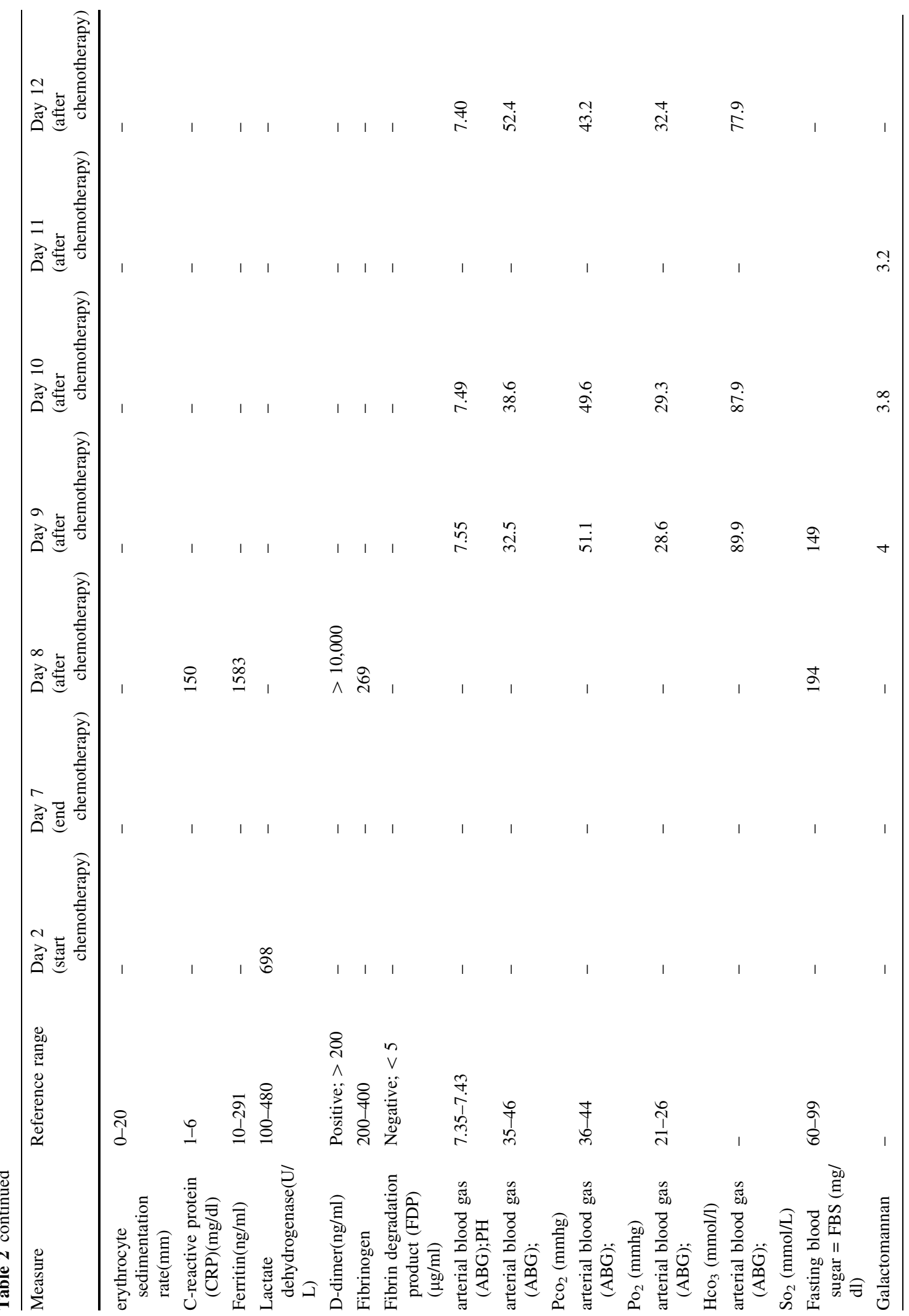




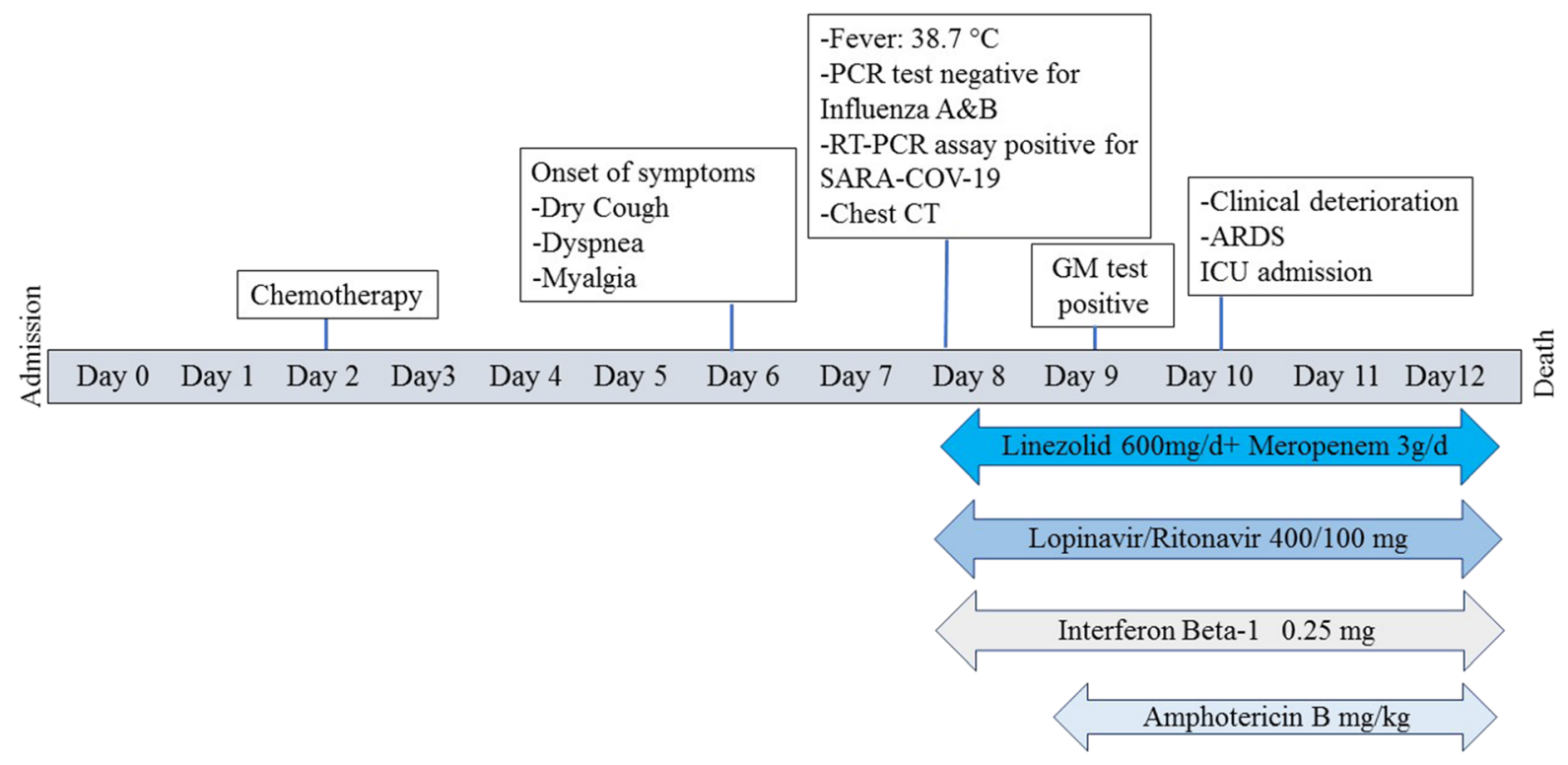

Fig. 2 Timeline representing the course of COVID-19 pneumonia in acute myeloid leukemia patient with probable aspergillosis

ventilation (NIPPV), and on hospital day 10, the patient was transferred to the ICU requiring endotracheal intubation and mechanical ventilation for refractory hypoxia despite NIPPV. Full laboratory blood analysis revealed white blood cells (WBC) $400 / \mu \mathrm{L}$ and C-reactive protein $165 \mathrm{mg} / \mathrm{dL}$. Serum galactomannan antigen (GM) test with Platelia Aspergillus ELISA kit (Bio-Rad) were 4, 3.8, 3 indexes on three consecutive days (Table 2). Chest-computed tomography (CT) revealed multiple nodular opacities with grand glass halo consisting of central and peripheral distribution (Fig. 1), and based on these results and the positive serum GM results, a diagnosis of probable invasive pulmonary aspergillosis with COVID-19 pneumonia was made. Therefore, intravenous liposomal amphotericin B $5 \mathrm{mg} / \mathrm{kg} /$ day was initiated. Despite antifungal and antibacterial therapy, the patient's condition continued to deteriorate, and the patient died the morning of day 12 due to respiratory and hemodynamic instability (Fig. 2).

\section{Discussion}

As of September 17, 2020, the COVID-19 pandemic due to SARS-CoV-2 has thus far killed over 23,808 people and infected over 413,149 individuals in Iran. Recent studies have shown that COVID-19 associated pulmonary aspergillosis (CAPA) may occur, especially in critically ill patients $[2,6]$. We report the first probable case of aspergillosis in an acute myeloid leukemia patient with COVID-19 associated with severe ARDS in Iran. Immediately after chemotherapy, the patient became neutropenic, interestingly the maximal reductions in neutrophil counts typically occur 6-14 days after conventional doses of anthracyclines, antifolates, and antimetabolites[9] . CT scan is an important imaging modality assisting in the diagnosis and management of patients with COVID19. IPA is a possible complication of critically ill patients with ARDS due to COVID-19 [1-4, 10]. Putative IPA was found in nearly $33 \%$ of mechanically ventilated patients with influenza or COVID-19 even in the absence of previous immune disorders [3, 10]. In previous outbreaks of SARS, proven invasive pulmonary aspergillosis were reported [11]. Serum GM screening detects a polysaccharide antigen found principally in the cells walls of Aspergillus species, and this assay is a sensitive diagnostic marker (70\%) in neutropenic patients $[2,8]$ and also positive in this case. As bronchoscopy generates aerosols that cause considerable risk to health care workers, and due to thrombocytopenia in this patient, it was not performed, and GM testing is a suitable diagnostic assay for IPA in lower respiratory tract specimens. The European Organization for Research and Treatment of 
Cancer/Mycoses Study Group (EORTC/MSG) criteria for invasive aspergillosis are appropriate for hematologic malignancy patients, and our patient was considered to have probable IPA based on an abnormal CT scan of the lungs and serum GM results, in addition to the typical COVID-19 attributed lesions [12]. In contrast, Alanio et al. reported a case of a patient with myeloma who presented with probable IPA with one nodule on chest X-rays in addition to the typical COVID-19 attributed lesions along with a positive bronchoalveolar lavage (BAL) culture, but a negative Aspergillus quantitative real-time PCR and serum galactomannan test [6]. In addition, Rutsaert et al. reported patient with AML who had developed IPA during chemotherapy with A. fumigatus growth in BAL culture, although a CT scan and histological examination were not available [13]. Due to challenges in the diagnosis of IPA in critically ill patients with COVID-19, clinicians should maintain a high level of suspicion for this invasive mycosis [1, 6]. Consequently, GM test is simple, and the specificity of an ODI-cutoff of $>1.0$ is about $90 \%$ to detect IPA [14]. Detection of GM has contributed substantially to the diagnosis of probable IA and is validated in a variety of patient groups, particularly with serial measurements [15-18]. Studies have suggested a sensitivity as high as $89 \%$ and a specificity of $92 \%$ in high-risk HSCT patients not receiving anti-mold prophylaxis, and a meta-analysis using an optical density index cutoff of $\geq 0.5$ showed a sensitivity of $78 \%$ and a specificity of $81 \%$. Serial assessments of GM may also offer prognostic value in predicting clinical outcomes [15-18]. The AML patient reported here had classical risk factors for IPA, and it is well known that viral infections, such as severe COVID-19 pneumonia, are associated with immune dysregulation (both T-helper cell 2 (Th2) and Th1, impaired cellmediated immune response and increase the risk for IPA [2, 19]. Despite antifungal therapy, the patient died due to respiratory distress and pulmonary failure. Our case report highlights that clinical suspicion and screening are needed to evaluate the prevalence of IPA in immunocompromised patients infected with COVID-19. Co-infection with Aspergillus may lead to poor outcomes, especially in hematological malignancies patients. Therefore, testing for the presence of Aspergillus in lower respiratory secretions and GM in consecutive serum samples in COVID-19 patients and early clinical suspicion of IPA with timely and targeted antifungal therapy according to national and international guidelines are highly recommended.

Acknowledgements This study was financially supported by a grant from the School of Medicine, Isfahan University of Medical Sciences, Isfahan, Iran (No. 198338) which we gratefully acknowledge. The authors acknowledge Prof. Nathan P. Wiederhold for his comments on the manuscript and for careful editing.

Funding This study was supported by grants from the Emergency special program for COVID-19 of Isfahan University of Medical Sciences, Isfahan, Iran project (No. 198338).

\section{Compliance with Ethical Standards}

Conflict of interest The authors report no conflicts of interest.

Informed Consent Informed consent was obtained from the patient for publication of this case report and accompanying image.

\section{References}

1. Rutsaert L, Steinfort N, Van Hunsel T, Bomans P, Naesens $\mathrm{R}$, Mertes H, et al. COVID-19-associated invasive pulmonary Aspergillosis. Ann Intensive Care. 2020;10(1):71.

2. Prattes J, Valentin T, Hoenigl M, Talakic E, Reisinger AC, Eller P. Invasive pulmonary Aspergillosis complicating COVID-19 in the ICU-A case report. Med Mycol Case Rep. 2020. https://doi.org/10.1016/j.mmcr.2020.05.001.

3. Salehi M, Ahmadikia K, Badali H, Khodavaisy S. Opportunistic fungal infections in the epidemic area of COVID19: a clinical and diagnostic perspective from Iran. Mycopathologia. 2020;185(4):607-611. https://doi.org/10.1007/ s11046-020-00472-7.

4. Song G, Liang G, Liu W. Fungal co-infections associated with global COVID-19 pandemic: a clinical and diagnostic perspective from China. Mycopathologia. 2020;185(4): 599-606.

5. Al-Hatmi AMS, Mohsin J, Al-Huraizi A, Khamis F. COVID-19 associated invasive candidiasis. J Infect. 2020;7: S0163-4453(20)30539-9.

6. Alanio A, Dellière S, Fodil S, Bretagne S, Mégarbane B. Prevalence of putative invasive pulmonary Aspergillosis in critically ill patients with COVID-19. Lancet Respir Med. 2020;8(6):e48-e4949.

7. Chen N, Zhou M, Dong X, Qu J, Gong F, Han Y, et al. Epidemiological and clinical characteristics of 99 cases of 2019 novel coronavirus pneumonia in Wuhan, China: a descriptive study. The Lancet. 2020;395(10223):507-13.

8. Meijer EF, Dofferhoff AS, Hoiting O, Buil JB, Meis JF. Azole-Resistant COVID-19-Associated Pulmonary Aspergillosis in an Immunocompetent Host: A Case Report. J Fungi. 2020;6(2):79.

9. Jameson JL, Fauci AS, Kasper DL, Hauser SL, Longo DL, Loscalzo J, editors. Harrison's principles of internal 
medicine, 20th edition. New York: McGraw Hill, Health Professions Division; 2018. p. 499.

10. Koehler P, Cornely OA, Böttiger BW, Dusse F, Eichenauer DA, Fuchs F, et al. COVID-19 associated pulmonary Aspergillosis. Mycoses. 2020;63(6):528-34.

11. Wang H, Ding Y, Li X, Yang L, Zhang W, Kang W. Fatal Aspergillosis in a patient with SARS who was treated with corticosteroids. N Engl J Med. 2003;349:507-8.

12. Donnelly JP, Chen SC, Kauffman CA, Steinbach WJ, Baddley JW, Verweij PE, et al. Revision and update of the consensus definitions of invasive fungal disease from the European Organization for Research and Treatment of Cancer and the Mycoses Study Group Education and Research Consortium. Clin Infect Dis. 2019.

13. Rutsaert L, Steinfort N, Van Hunsel T, Bomans P, Naesens $\mathrm{R}$, Mertes H, et al. COVID-19-associated invasive pulmonary Aspergillosis. Ann Intensive Care. 2020;10(1):71. https://doi.org/10.1186/s13613-020-00686-4.

14. Lahmer T, Rasch S, Spinner C, Geisler F, Schmid RM, Huber W. Invasive pulmonary aspergillosis in severe COVID-19 pneumonia. Clin Microbiol Infect. 2020; 2,S1198-743X(20)30309-8.

15. Duarte RF, Sánchez-Ortega I, Cuesta I, Arnan M, Patino B, et al. Serum galactomannan-based early detection of invasive Aspergillosis in hematology patients receiving effective antimold prophylaxis. Clin Infect Dis. 2014;59:1696-702.

16. Marr KA, Balajee SA, McLaughlin L, Tabouret M, Bentsen $\mathrm{C}$, Walsh TJ, et al. Detection of galactomannan antigenemia by enzyme immunoassay for the diagnosis of invasive Aspergillosis: variables that affect performance. J Infect Dis. 2004;190(3):641-9.

17. Maertens J, Theunissen K, Verbeken E, Lagrou K, Varhaegen J, Boogaerts M, et al. Prospective clinical evaluation of lower cut-off for galactomannan detection in adult neutropenic cancer patients and haematological stem cell transplant recipients. Br J Haematol. 2004;126(6):852-60.

18. Leeang MM, Debets-Ossenkopp YJ, Visser CE, Scholten MPJR, Hooft L, Bijlmer AH, et al. Galactomannan detection for invasive Aspergillosis in immunocompromized patients. Cochrane Database Syst Rev. 2008;8(4):CD007394.

19. Verweij PE, Gangneux J-P, Bassetti M, Brüggemann RJ, Cornely OA, Koehler P, et al. Diagnosing COVID-19-associated pulmonary Aspergillosis. Lancet Microbe. 2020;1(2):e53-e5.

Publisher's Note Springer Nature remains neutral with regard to jurisdictional claims in published maps and institutional affiliations. 